OPEN ACCESS

Edited by:

Michele Papa

University of Campania Luigi

Vanvitelli, Italy

Reviewed by:

Federico Herrera,

New University of Lisbon, Portugal

Byoung Dae Lee,

Kyung Hee University, South Korea

*Correspondence:

Ji-Eun Kim

jieunkim@hallym.ac.kr

Tae-Cheon Kang

tckang@hallym.ac.kr

Received: 11 April 2019 Accepted: 09 July 2019 Published: 24 July 2019

Citation: Kim J-E and Kang T-C (2019) PKC, AKT and ERK1/2-Mediated Modulations of PARP1, NF- $\kappa$ B and PEA15 Activities Distinctly Regulate Regional Specific Astroglial

Responses Following Status Epilepticus.

Front. Mol. Neurosci. 12:180. doi: 10.3389/fnmol.2019.00180

\section{PKC, AKT and ERK1/2-Mediated Modulations of PARP1, NF- $\kappa$ B and PEA15 Activities Distinctly Regulate Regional Specific Astroglial Responses Following Status Epilepticus}

\author{
Ji-Eun Kim* and Tae-Cheon Kang* \\ Department of Anatomy and Neurobiology, Institute of Epilepsy Research, College of Medicine, Hallym University, \\ Chuncheon, South Korea
}

Status epilepticus (SE, a prolonged seizure activity) leads to reactive astrogliosis and astroglial apoptosis in the regional specific manners, independent of hemodynamics. Poly(ADP-ribose) polymerase-1 (PARP1) activity is relevant to these distinct astroglial responses. Since various regulatory signaling molecules beyond PARP1 activity may be involved in the distinct astroglial response to SE, it is noteworthy to explore the roles of protein kinases in PARP1-mediated reactive astrogliosis and astroglial apoptosis following SE, albeit at a lesser extent. In the present study, inhibitions of protein kinase $C$ (PKC), AKT and extracellular signal-related kinases 1/2 (ERK1/2), but not calcium/calmodulin-dependent protein kinase II (CaMKII), attenuated CA1 reactive astrogliosis accompanied by reducing PARP1 activity following SE, respectively. However, inhibition of AKT and ERK1/2 deteriorated SE-induced dentate astroglial loss concomitant with the diminished PARP1 activity. Following SE, PKC- and AKT inhibitors diminished phosphoprotein enriched in astrocytes of $15 \mathrm{kDa}$ (PEA15)-S104 and -S116 phosphorylations in CA1 astrocytes, but not in dentate astrocytes, respectively. Inhibitors of PKC, AKT and ERK1/2 also abrogated SE-induced nuclear factor-кB (NF-kB)-S311 and -S468 phosphorylations in CA1 astrocytes. In contrast, both AKT and ERK1/2 inhibitors enhanced NF-kB-S468 phosphorylation in dentate astrocytes. Furthermore, PARP1 inhibitor aggravated dentate astroglial loss following SE. AKT inhibition deteriorated dentate astroglial loss and led to CA1 astroglial apoptosis following SE, which were ameliorated by AKT activation. These findings suggest that activities of PARP1, PEA15 and NF-KB may be distinctly regulated by PKC, AKT and ERK1/2, which may be involved in regional specific astroglial responses following SE.

Keywords: 3CAI, BIM, epilepsy, KN-93, PJ-34, SC79, seizure, U0126 


\section{INTRODUCTION}

Astrocytes are the most abundant glial cells, which participate in a wide variety of complex and essential functions including the maintenance of neuronal excitability (Anderson and Swanson, 2000; Mazzanti et al., 2001) and the homeostasis of extracellular environment as well as metabolism in the brain (Kasischke et al., 2004; Simard and Nedergaard, 2004; Takano et al., 2006). Following various insults, astrocytes show reactive astrogliosis, which inhibits dendritic and axonal remodeling in neuronal circuits, and releases various growth and trophic factors regulating synaptogenesis, neurogenesis, and neuroinflammation after brain injury (Horner and Gage, 2000; Panickar and Norenberg, 2005; Rossi et al., 2007; Shibuya, 2009).

Accompanied by reactive astrogliosis, status epilepticus (SE, a prolonged seizure activity) results in acute and devastating astroglial loss, which is characterized by a pattern of selective vulnerability (Schmidt-Kastner and Ingvar, 1994, 1996). In the hippocampus, SE leads to apoptosis of astrocytes in the molecular layer of the dentate gyrus (referred as dentate astrocytes below; Kang et al., 2006; Kim et al., 2008, 2014, 2017). This SE-induced astroglial apoptosis is relevant to poly(ADP-ribose) polymerase-1 (PARP1) degradation (Kim et al., 2014). Unlike the molecular layer of the dentate gyrus, PARP1 activation is involved in reactive gliosis of astrocyte in the CA1 region (referred as CA1 astrocytes below) where astroglial apoptosis is undetected (Kang et al., 2006; Kim et al., 2014). Basically, PARP1 repairs DNA damage following various injuries. Thus, PARP1 degradation and/or its cleavage lead to apoptosis (Kaufmann et al., 1993; Lazebnik et al., 1994). Since PARP1 utilizes $\mathrm{NAD}^{+}$to form poly(ADPribose) polymers (PAR), PARP1 hyperactivation also leads to $\mathrm{NAD}^{+}$depletion and the subsequent failure of bioenergetics that promotes necrotic cell death (Ha and Snyder, 1999; Ying et al., 2002).

Phosphoprotein enriched in astrocytes of $15 \mathrm{kDa}$ (PEA15) is a small phosphoprotein, which is abundantly expressed in astrocytes (Danziger et al., 1995), and protects astrocytes from apoptosis (Estellés et al., 1999; Kitsberg et al., 1999). PEA15 functionality is controlled by phosphorylations: Protein kinase C (PKC) phosphorylates serine (S) 104 site. Calcium/calmodulin-dependent protein kinase II (CaMKII) or AKT preferentially phosphorylate S116 site (Araujo et al., 1993; Estellés et al., 1996; Kubes et al., 1998). Phosphorylated PEA15 accelerates nuclear extracellular signal-related kinases $1 / 2(E R K 1 / 2)$ translocation that activates astroglial proliferation and up-regulation of glial fibrillary acidic protein (GFAP) expression, which are hallmarks of reactive astrogliosis (Liu et al., 2004; Krueger et al., 2005; Meini et al., 2008; Kim and Kang, 2018). Recently, we have reported that the reduced PEA15 expression and its S116 phosphorylation are involved in astroglial apoptosis, while its S104 phosphorylation is up-regulated in reactive CA1 astrocytes following SE (Park and Kang, 2018). Interestingly, protein kinases for PEA15 phosphorylations also reciprocally influence PARP1 activity. For example, PKC inhibits PARP1 (Hegedus et al., 2008), while ERK1/2 activates PARP1 (Kauppinen et al.,
2006; Cohen-Armon et al., 2007; Mizuguchi et al., 2011). CaMKII also affects PARP1 enzyme activity (Midorikawa et al., 2006; Goebel, 2009), and PARP1 increases AKT activity (Gerace et al., 2012). Furthermore, both PARP1 and PEA15 regulates

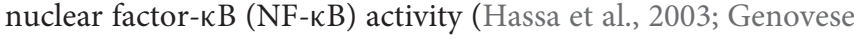
et al., 2005; Stilmann et al., 2009; Wakita et al., 2016), which is is also involved in reactive astrogliosis (Morga et al., 2009). Therefore, it is noteworthy to explore the roles of various kinases in activities of PARP1, PEA15 and NF- $\mathrm{B}$ during reactive astrogliosis or astroglial apoptosis following $\mathrm{SE}$, which remain elusive.

Here, we demonstrate that bisindolylmaleimide (BIM, a PKC inhibitor), 3-chloroacetyl-indole (3CAI, an AKT inhibitor) and U0126 (an ERK1/2 inhibitor), but not KN-93 (a CaMKII inhibitor), attenuated CA1 reactive astrogliosis accompanied by reducing PARP1 activity following SE, respectively. However, 3CAI and U0126 deteriorated SE-induced dentate astroglial loss concomitant with the diminished PARP1 activity. BIM and 3CAI attenuated SE-induced PEA15-S104 and -S116 phosphorylations in CA1 astrocytes, respectively. U0126 and KN-93 did not affect PEA15 phosphorylations in CA1 astrocytes. BIM, 3CAI and U0126 also abrogated SE-induced NF- $\kappa$ B-S311 phosphorylation in CA1 astrocytes. In contrast, 3CAI and U0126 enhanced NF- $\mathrm{B}-\mathrm{S} 468$ phosphorylation in dentate astrocytes. Furthermore, PJ-34 (a PARP1 inhibitor) aggravated dentate astroglial loss following SE. 3CAI deteriorated dentate astroglial loss and led to CA1 astroglial apoptosis, which was ameliorated by SC79 (an AKT activator). These findings suggest that PKC, AKT and ERK1/2 may distinctly regulate activities of PARP1, PEA15 and NF- $\kappa$ B in regional specific astroglial apoptosis and reactive astrogliosis following $\mathrm{SE}$.

\section{MATERIALS AND METHODS}

\section{Experimental Animals and Chemicals}

All animal experimental procedures and protocols were approved by the Institutional Animal Care and Use Committee of the Hallym University (Chuncheon, South Korea). Adult male Sprague-Dawley (SD) rats weighting 250-280 g, were purchased from Daehan Biolink (South Korea). Rats were housed under controlled environmental conditions $\left(23-25^{\circ} \mathrm{C}, 12 \mathrm{~h}\right.$ light/dark cycle) with free access to water and standard laboratory food. All reagents were obtained from Sigma-Aldrich (USA) unless otherwise noted.

\section{Intracerebroventricular Drug Infusion}

Under Isoflurane anesthesia $\left(1 \%-2 \%\right.$ in $\mathrm{O}_{2}$ and $\left.\mathrm{N}_{2} \mathrm{O}\right)$, animals were stereotaxically implanted a brain infusion kit 1 (Alzet, Cupertino, CA, USA) into the lateral ventricle (1 $\mathrm{mm}$ posterior; $1.5 \mathrm{~mm}$ lateral; $-3.5 \mathrm{~mm}$ depth; flat skull position with bregma as reference). Thereafter, an infusion kit was connected to an osmotic pump (1007D, Alzet, Cupertino, CA, USA) containing: (1) vehicle; (2) bisindolylmaleimide (BIM, a PKC inhibitor, $25 \mu \mathrm{M}$ ); (3) 3-chloroacetyl-indole (3CAI, an AKT inhibitor, $25 \mu \mathrm{M}$ ); (4) U0126 (an ERK1/2 inhibitor, $25 \mu \mathrm{M}$ ); (5) KN-93 (a CaMKII inhibitor, $25 \mu \mathrm{M}$, Santa Cruz, CA, USA); (6) PJ-34 (PARP inhibitor VIII, $3 \mu \mathrm{M}$, Merck, 
Germany); and (7) SC79 (an AKT activator, $25 \mu \mathrm{M}$ ). In a pilot study and our previous studies (Kim et al., 2014; Kim and Kang, 2018; Park and Kang, 2018), each compound treatment did not show behavioral and neurological defects in animals and did not affect seizure threshold in response to pilocarpine. Three days after surgery, animals were used for SE induction.

\section{SE Induction}

SE was induced by a single dose $(30 \mathrm{mg} / \mathrm{kg})$ of pilocarpine in rats pretreated ( $24 \mathrm{~h}$ before pilocarpine injection) with $127 \mathrm{mg} / \mathrm{kg}$ lithium chloride, as previously described (Kim et al., 2014; Kim and Kang, 2018; Park and Kang, 2018). Before pilocarpine injection, animals were given atropine methylbromide $(5 \mathrm{mg} / \mathrm{kg}$ i.p.) to block the peripheral effect of pilocarpine. Two hours after SE, animals received diazepam (10 $\mathrm{mg} / \mathrm{kg}$, i.p. $)$ to terminate SE. As controls, rats were treated with saline instead of pilocarpine.

\section{Tissue Processing}

Three days after SE, animals were deeply anesthetized with urethane anesthesia (1.5 g/kg, i.p.) and immediately cardiacperfused with phosphate-buffered saline (PBS, $\mathrm{pH}$ 7.4) followed by $4 \%$ paraformaldehyde in $0.1 \mathrm{M}$ phosphate buffer ( $\mathrm{PB}, \mathrm{pH} 7.4$ ). After perfusion, brains were quickly removed and post-fixed in the $4 \%$ paraformaldehyde and cryoprotected by $30 \%$ sucrose overnight. Thereafter, the tissues were sectioned with a cryostat at $30 \mu \mathrm{m}$ and consecutive sections were collected in six-well plates containing PBS.

\section{TUNEL Staining}

According to the manufacturer's protocol, TUNEL staining was performed using TUNEL apoptosis detection kit (Upstate, Lake Placid, NY, USA). Following the TUNEL reaction, double fluorescent staining was performed (see below).

\section{Immunofluorescent Study}

Sections were incubated in a mixture of appropriate primary antibodies (Table 1) in PBS containing $0.3 \%$ Triton X-100 overnight at room temperature. For triple immunofluorescent study, we used M.O.M. kit (Vector, USA, \#BMK-2202) according to the manufacturer's protocol. After washing three times for 10 min with PBS, the sections were also incubated in a mixture of AMCA- (or FITC-) and Cy3-conjugated secondary antisera (or streptavidin, 1:250, Amersham, USA) for $2 \mathrm{~h}$ at room temperature. For negative control, the hippocampal tissues were incubated with pre-immune serum instead of primary antibody. All images were captured using an Axio Imager M2 microscope and AxioVision Rel. 4.8 software (Carl Zeiss Korea, Seoul, South Korea).

\section{Statistical Analysis}

For quantitative analysis of fluorescent intensity, sections (15 sections per each animal) were viewed through a microscope connected via CCD camera (Carl Zeiss Korea). Thereafter, fluorescent intensity measurements were represented as the number of a 256-gray scale using AxioVision Rel. 4.8 software (Carl Zeiss Korea). Intensity values were corrected by subtracting
TABLE 1 | Primary antibodies used in the present study.

\begin{tabular}{lllc}
\hline Antigen & Host & $\begin{array}{l}\text { Manufacturer } \\
\text { (catalog number) }\end{array}$ & Dilution used \\
\hline GFAP & Mouse & Millipore (\#MAB3402) & $1: 500$ \\
NF-kB RelA p65-S311 & Rabbit & Abcam (ab194926) & $1: 50$ \\
NF-kB RelA p65-S468 & Rabbit & Abcam (ab31473) & $1: 50$ \\
PAR & Mouse & Enzo (ALX-804-220) & $1: 100$ \\
PARP1 & Rabbit & Abcam (ab32138) & $1: 100$ \\
PEA15-S104 & Mouse & Trevigen (4338-MC-50) & $1: 100$ \\
& Rabbit & Antibodies-online & $1: 200$ \\
PEA15-S116 & & (ABIN744683) & $1: 200$ \\
& Rabbit & Antibodies-online & \\
\hline
\end{tabular}

the average values of background noise obtained from five image inputs. The optical density was then standardized by setting the threshold levels. In addition, two different investigators performed TUNEL-positive cell counts. All data obtained from the quantitative measurements were tested for the normality and equality of variance. Thereafter, data were analyzed by one-way analysis of variance (ANOVA) coupled with Bonferroni's post hoc test for multiple comparisons. Values are presented as mean \pm standard error of the mean (SEM). Differences were considered as significant for $p<0.05$.

\section{RESULTS}

\section{Effects of Kinase Inhibitors on Astroglial PARP1 Activity Following SE}

First, we validated the characteristics of various kinase inhibitors in regional specific astroglial responses to SE. As compared to control animals, SE led to the increases in PARP1 expression and PAR level in CA1 astrocytes ( $p<0.05$ vs. control; one-way ANOVA, $n=7$, respectively; Figures 1A,B). As compared to vehicle, BIM (a PKC inhibitor) attenuated the increased PARP1 expression and PAR level in CA1 astrocytes following $\mathrm{SE}(p<0.05$ vs. vehicle, one-way ANOVA, $n=7$, respectively; Figures 1A,B). 3CAI (an AKT inhibitor) diminished PARP1 expression and PAR level in CA1 astrocytes more than BIM ( $p<0.05$ vs. vehicle and BIM, one-way ANOVA, $n=7$, respectively; Figures 1A,B). U0126 (an ERK1/2 inhibitor) reduced PAR level without altering PARP1 expression in CA1 astrocytes ( $p<0.05$ vs. vehicle; one-way ANOVA, $n=7$; Figures 1A,B). However, KN-93 (a CaMKII inhibitor) did not affect the increased PARP1 expression and PAR level in CA1 astrocytes following SE (Figures 1A,B).

Consistent with our previous studies (Kang et al., 2006; Kim et al., 2008, 2017), SE led to massive loss of dentate astrocytes, accompanied by reducing PARP1 expression and PAR level $(p<0.05$ vs. vehicle, one-way ANOVA, $n=7$, respectively; Figures 1A,C). U0126 and 3CAI deteriorated dentate astroglial loss concomitant with decreasing PARP1 expression ( $p<0.05$ vs. vehicle, one-way ANOVA, $n=7$, respectively; Figures 1A,C), while BIM and $\mathrm{KN}-93$ did not affect SE-induced dentate astroglial loss without altering PARP1 expression and PAR level. These findings indicate that PKC, AKT and ERK1/2, but not CaMKII, may regulate PARP1-mediated CA1 reactive 


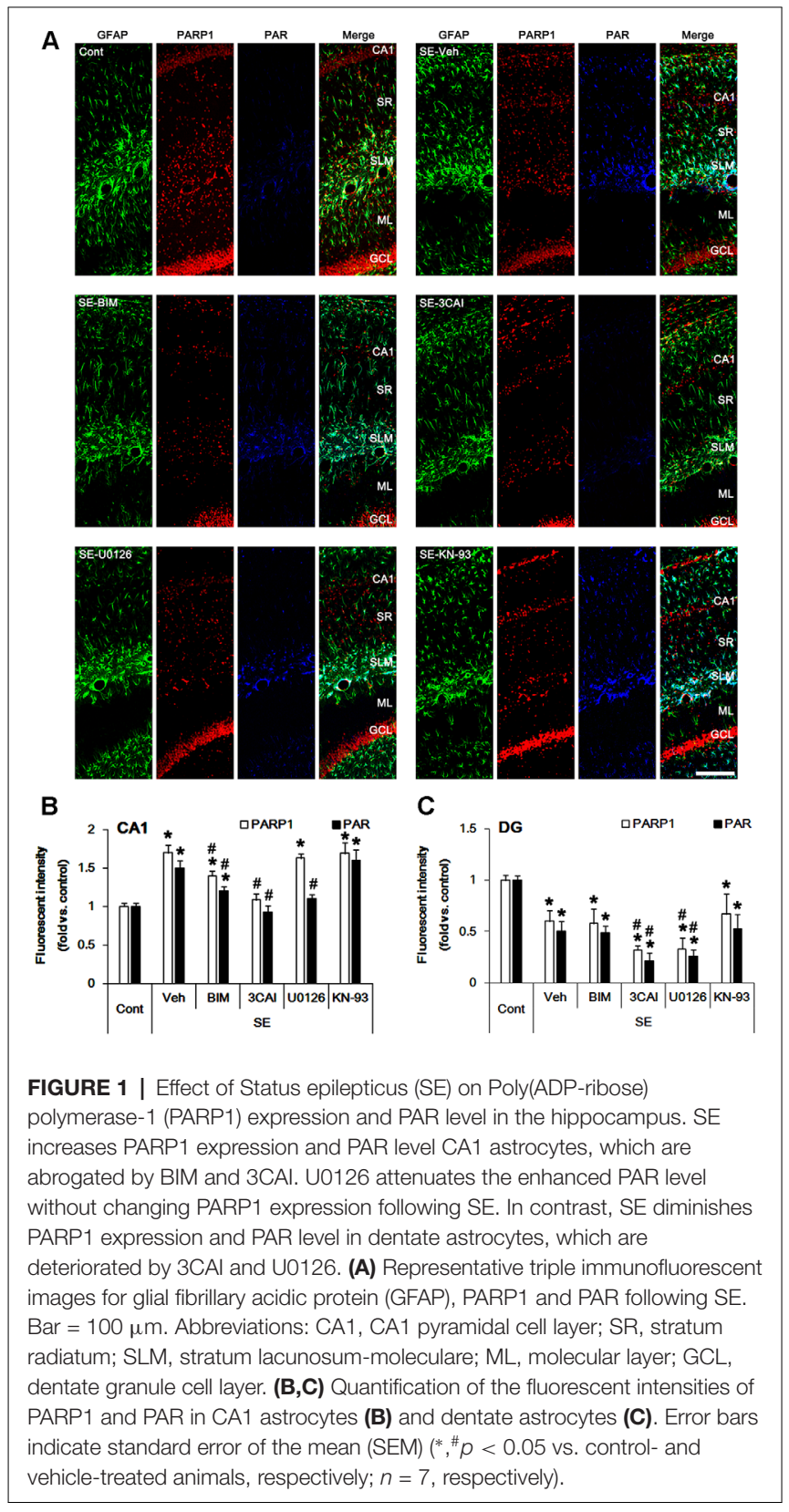

astrogliosis and that AKT and ERK1/2 may be also relevant to SE-induced dentate astroglial loss.

\section{Effects of Kinase Inhibitors on Astroglial PEA15 Phosphorylations Following SE}

PEA15 expression and its phosphorylations are involved in astroglial response to SE (Park and Kang, 2018). Therefore, we evaluated the effects of kinase inhibitors on PEA15-S104 and -S116 phosphorylations in astrocytes following SE. In control animals, PEA15-S104 immunoreactivity was predominantly observed in CA1 astrocytes (Figure 2A). PEA15-S104 phosphorylation was up-regulated in reactive CA1 astrocytes ( $p<0.05$ vs. control animals, one-way ANOVA, $n=7$; Figures 2A,B). BIM down-regulated PEA15-S104
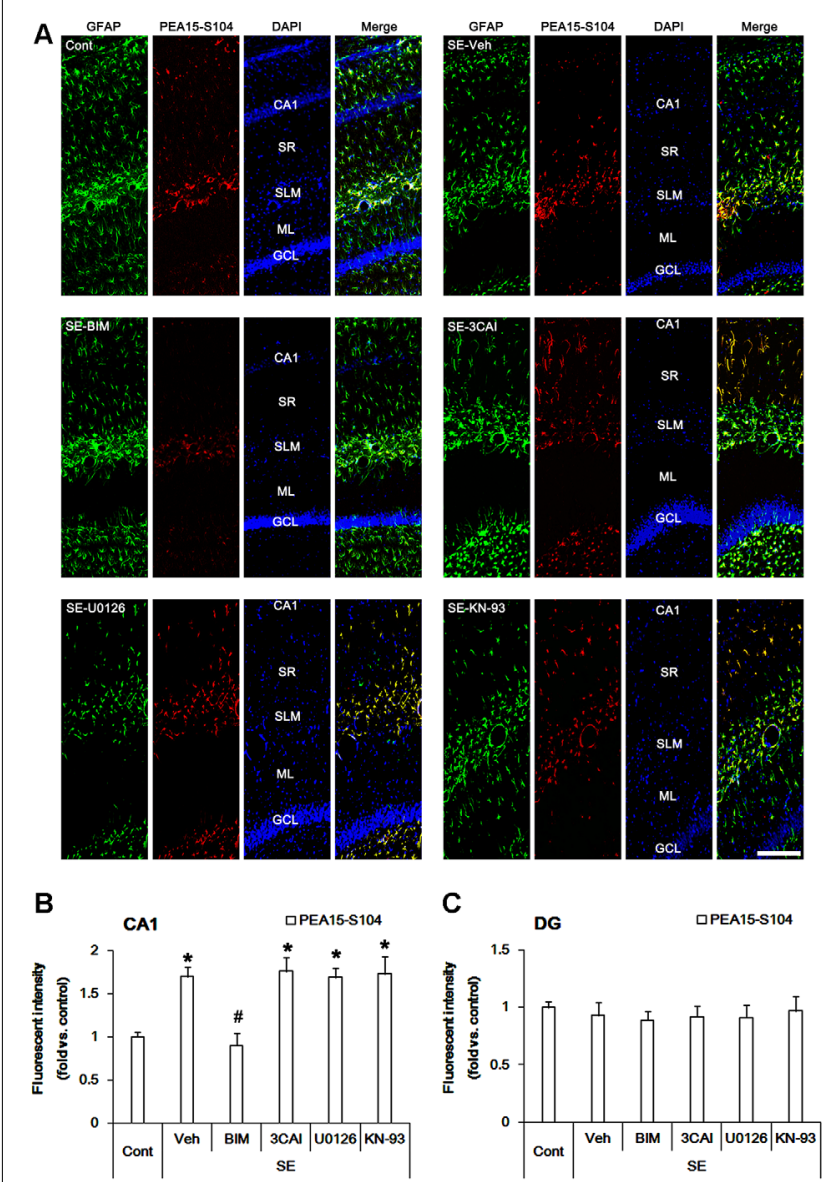

FIGURE 2 | Alterations in PEA15-S104 phosphorylation in the hippocampus following SE. SE enhances PEA15-S104 phosphorylation in CA1 astrocytes, which is reversed by BIM, but not 3CAI, U0126 and KN-93. SE does not influence PEA15-S104 phosphorylation in dentate astrocytes, which is unaffected by BIM, 3CAI, U0126 and KN-93. (A) Representative immunofluorescent images for GFAP and PEA15-S104 phosphorylation following SE. Bar $=100 \mu \mathrm{m}$. Abbreviations: CA1, CA1 pyramidal cell layer; $\mathrm{SR}$, stratum radiatum; SLM, stratum lacunosum-moleculare; ML, molecular layer; GCL, dentate granule cell layer. (B,C) Quantification of the fluorescent intensities of PEA15-S104 phosphorylation in CA1 astrocytes (B) and dentate astrocytes (C). Error bars indicate SEM $\left({ }^{*},{ }^{\#} p<0.05\right.$ vs. control- and vehicle-treated animals, respectively; $n=7$, respectively).

phosphorylation level in reactive CA1 astrocytes $(p<0.05$ vs. vehicle, one-way ANOVA, $n=7$; Figures 2A,B). 3CAI, U0126 and KN-93 did not affect PEA15-S104 phosphorylation in reactive CA1 astrocytes. As compared to control animals, PEA15-S104 phosphorylation level was unaltered in dentate astrocytes following SE (Figures 2A,C). BIM, 3CAI, U0126 and KN-93 did not influence PEA15-S104 phosphorylation level in dentate astrocytes (Figures 2A,C). These findings indicate that PKC-mediated PEA15-S104 phosphorylation may be relevant to CA1 reactive astrogliosis, while PEA15-S104 phosphorylation may not be involved in degeneration of dentate astrocytes following SE.

In control animals, PEA15-S116 immunoreactivity was detected in CA1 astrocytes and dentate astrocytes. In contrast to S104 phosphorylation, PEA15-S116 phosphorylation was 


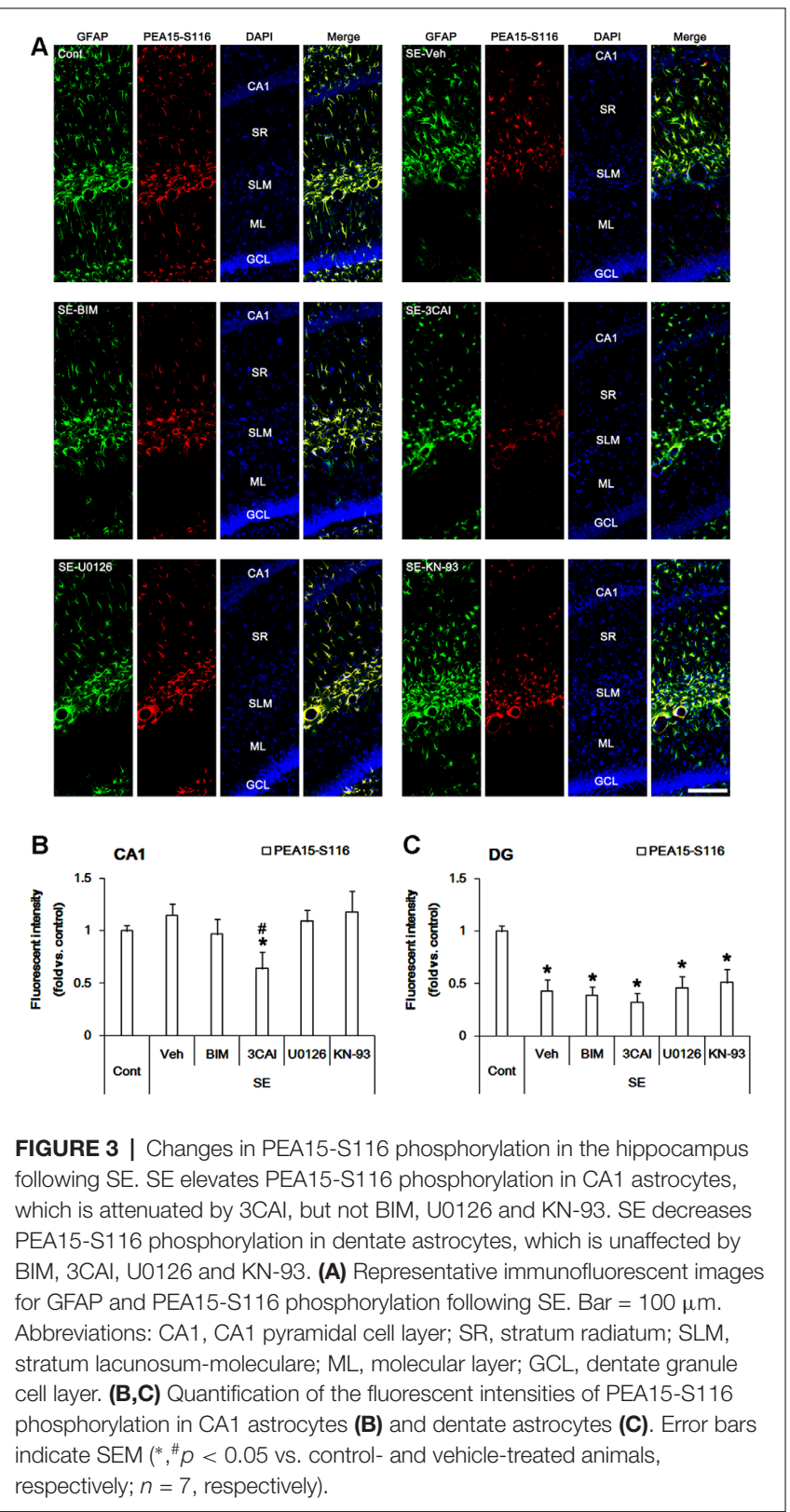

unaltered in CA1 astrocytes following SE (Figures 3A,B). 3CAI reduced PEA15-S116 phosphorylation in reactive CA1 astrocytes $(p<0.05$ vs. vehicle, one-way ANOVA, $n=7$; Figures 3A,B), while BIM, U0126 and KN-93 did not influence PEA15-S116 phosphorylation following SE. Unlike CA1 astrocytes, PEA15-S116 phosphorylation was reduced in dentate astrocytes following SE $(p<0.05$ vs. control animals, one-way ANOVA, $n=7$; Figures 3A,C). BIM, 3CAI, U0126 and KN-93 did not affect the reduction in PEA15-S116 phosphorylation in dentate astrocytes following SE. Considering CaMKII- and AKT-mediated PEA15-S116 phosphorylation (Araujo et al., 1993; Estellés et al., 1996; Kubes et al., 1998), these findings indicate that AKT, but not CaMKII, may play a role in the maintenance of PEA15-S116 phosphorylation in $\mathrm{CA} 1$ reactive astrogliosis following $\mathrm{SE}$.
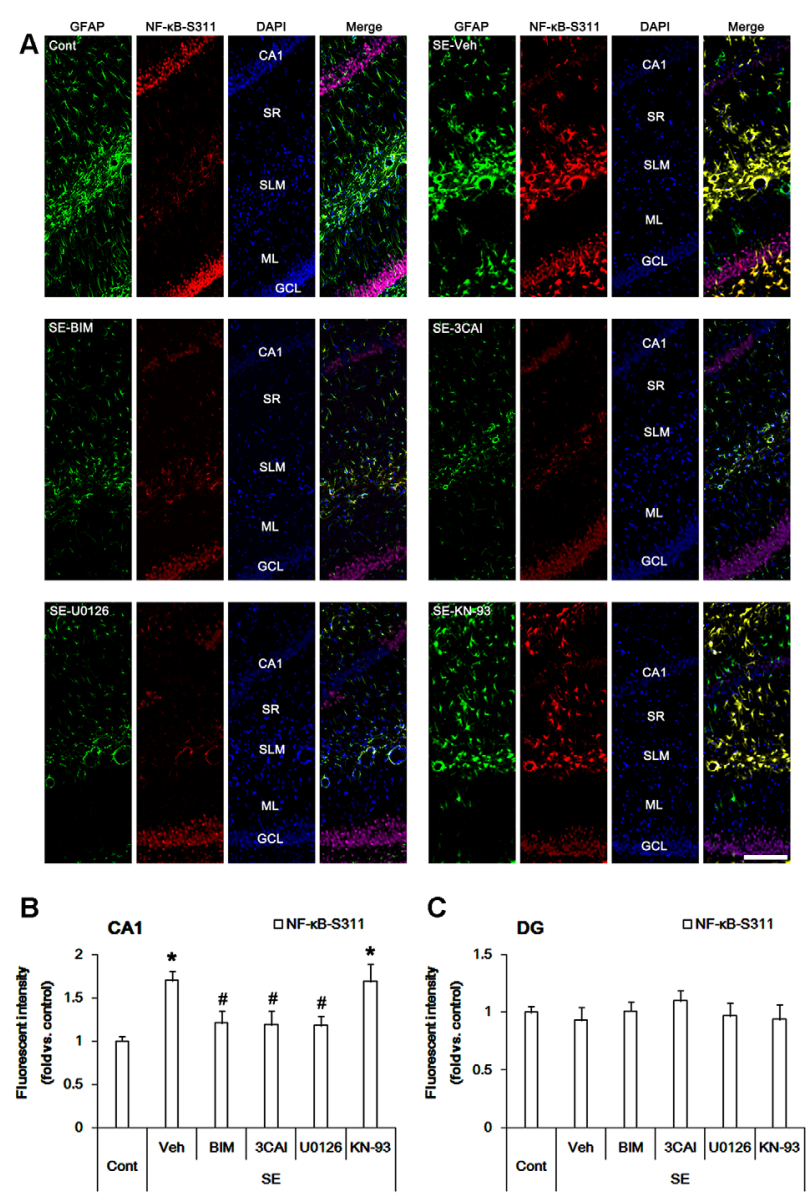

FIGURE 4 | Alterations in NF-кB-S311 phosphorylation in the hippocampus following SE. SE increases NF-кB-S311 phosphorylation in CA1 astrocytes, which is attenuated by BIM, 3CAI, and U0126, but not KN-93. SE does not influence NF-кB-S311 phosphorylation in dentate astrocytes. In addition, NF-кB-S311 phosphorylation is unaffected by BIM, 3CAI, U0126 and KN-93. (A) Representative immunofluorescent images for GFAP and PEA15-S116 phosphorylation following SE. Bar $=100 \mu \mathrm{m}$. Abbreviations: CA1, CA1 pyramidal cell layer; SR, stratum radiatum; SLM, stratum lacunosum-moleculare; ML, molecular layer; GCL, dentate granule cell layer. $\mathbf{( B , C )}$ Quantification of the fluorescent intensities of

NF-kB-S311 phosphorylation in CA1 astrocytes (B) and dentate astrocytes (C). Error bars indicate SEM (*, ${ }^{*} p<0.05$ vs. control- and vehicle-treated animals, respectively; $n=7$, respectively).

\section{Effects of Kinase Inhibitors on Astroglial NF-кB Phosphorylations Following SE}

Since p65 RelA NF- $\kappa$ B (referred as NF- $\kappa$ B below) phosphorylation is also involved in reactive astrogliosis (Morga et al., 2009), we investigated the effects on protein kinase inhibitors on NF- $\mathrm{kB}-\mathrm{S} 311$ and -S468 phosphorylations that modulate its optimal activity (Viatour et al., 2005). Consistent with our previous studies (Kim et al., 2013), NF-кB-S311 phosphorylation was observed in a few CA1 astrocytes (Figure $\mathbf{4 A}$ ). Following SE, NF-кBS311 phosphorylation was increased in CA1 astrocytes $(p<0.05$ vs. control animals, one-way ANOVA, $n=7$; Figures 4A,B). BIM, 3CAI and U0126, but not KN-93, 

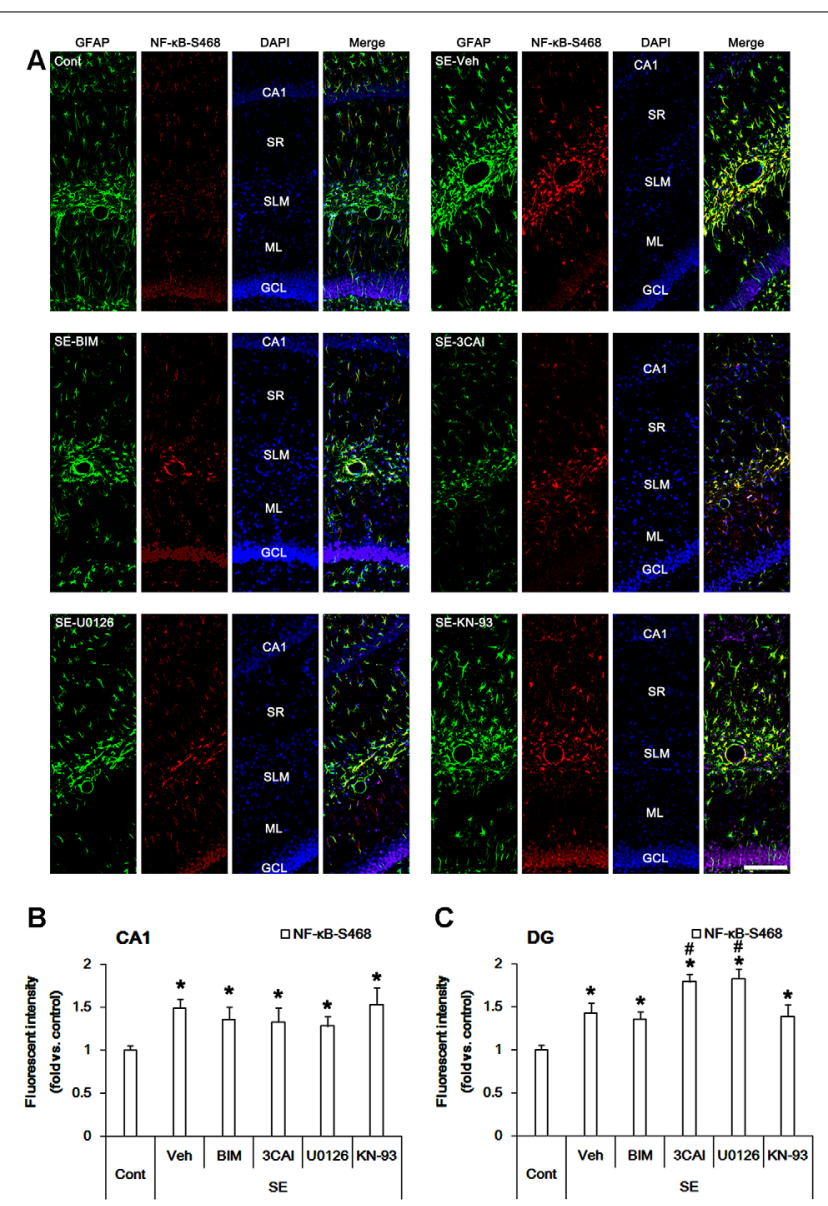

FIGURE 5 | Effect of SE on NF-кB-S468 phosphorylation in the hippocampus. SE increases NF-кB-S468 phosphorylation in CA1 astrocytes, which is unaffected by BIM, 3CAI, U0126 and KN-93. SE also elevates NF-кB-S468 phosphorylation in dentate astrocytes, which is enhanced by 3CAl and U0126. (A) Representative immunofluorescent images for GFAP and NF-кB-S468 phosphorylation following SE. Bar $=100 \mu \mathrm{m}$. Abbreviations: CA1, CA1 pyramidal cell layer; SR, stratum radiatum; SLM, stratum lacunosum-moleculare; ML, molecular layer; GCL, dentate granule cell layer. (B,C) Quantification of the fluorescent intensities of

NF-кB-S468 phosphorylation in CA1 astrocytes (B) and dentate astrocytes

(C). Error bars indicate SEM (*, ${ }^{*} p<0.05$ vs. control- and vehicle-treated

animals, respectively; $n=7$, respectively).

attenuated the increased NF- $\kappa \mathrm{B}-\mathrm{S} 311$ phosphorylation in CA1 astrocytes $(p<0.05$ vs. vehicle, one-way ANOVA, $n=7$, respectively; Figures $4 \mathbf{A}, \mathbf{B})$. As compared to control animals, NF- $\kappa \mathrm{B}-\mathrm{S} 311$ phosphorylation was unaltered in dentate astrocytes following SE (Figures 4A,C). BIM, 3CAI, U0126 and KN-93 did not influence NF- $\kappa$ B-S311 phosphorylation level in dentate astrocytes (Figures 4 A,C). These findings suggest that PARP1 activation and NF- $\kappa \mathrm{B}-\mathrm{S} 311$ phosphorylation may play an important role in $\mathrm{CA} 1$ reactive astrogliosis.

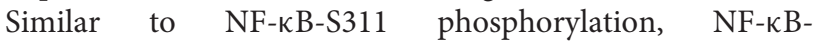
S468 positivity was increased in CA1 astrocytes following SE ( $p<0.05$ vs. vehicle, one-way ANOVA, $n=7$, respectively; Figures 5A,B). However, BIM, 3CAI, U0126 and KN-93 did not affect NF- $\mathrm{B}-\mathrm{S} 468$ phosphorylation in CA1 astrocytes
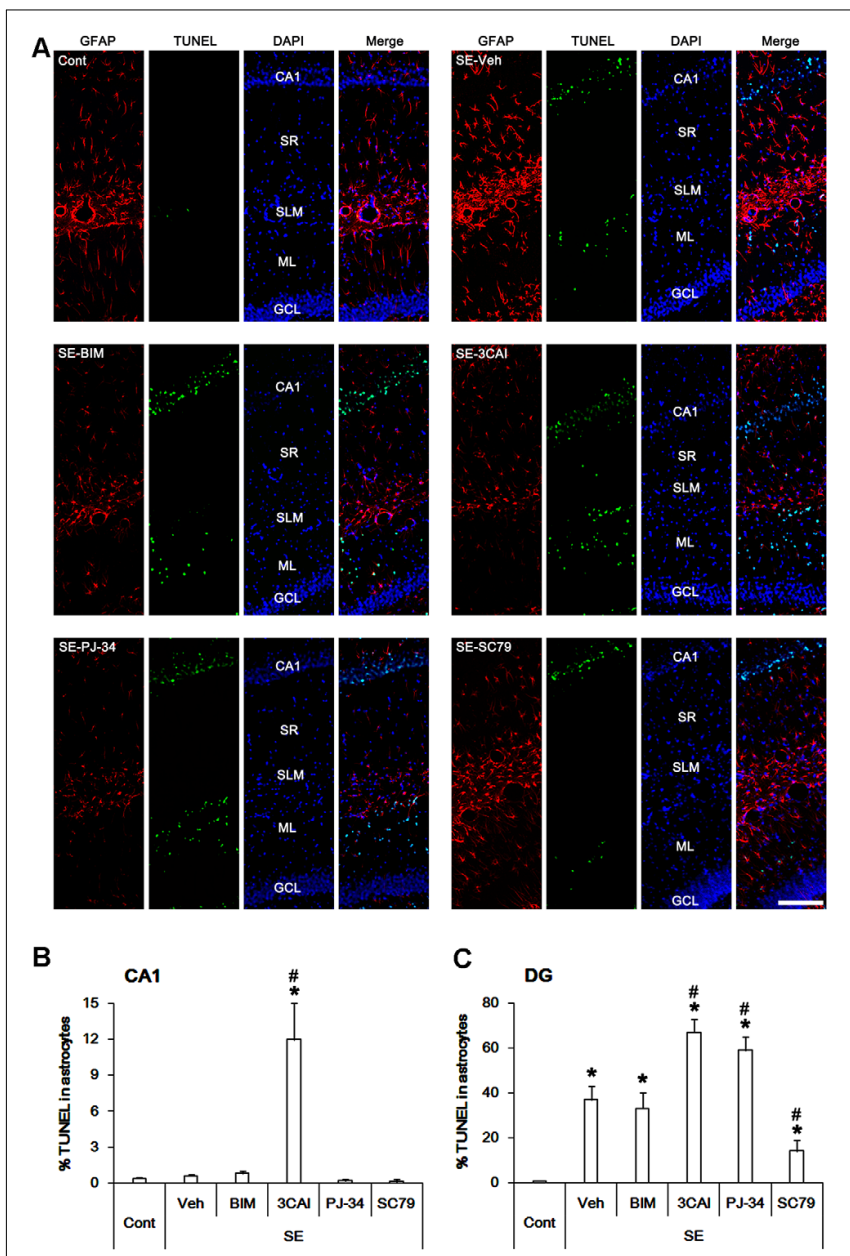

FIGURE 6 | Astroglial apoptosis in the hippocampus following SE. SE does not induce apoptosis in CA1 astrocytes. 3CAl leads to CA1 astroglial apoptosis following SE. However, SE result in apoptotic degeneration in dentate astrocytes, which is deteriorated by 3CAI and PJ-34.

SC79 ameliorates dentate astroglial apoptosis following SE. (A)

Representative immunofluorescent images for GFAP and TUNEL following SE. (B,C) Quantification of the percentage of TUNEL positive astrocytes in CA1 astrocytes (B) and dentate astrocytes (C). Error bars indicate SEM ${ }^{*},{ }^{\#} p<0.05$ vs. control- and vehicle-treated animals, respectively; $n=7$, respectively).

(Figures 5A,B). SE also increased NF- $\mathrm{B}-\mathrm{S} 468$ phosphorylation in dentate astrocytes. 3CAI and U0126 enhanced NF-кBS468 phosphorylation in these cells following SE $(p<0.05$ vs. vehicle, one-way ANOVA, $n=7$, respectively; Figures 5A,C), while BIM and KN-93 did not affect it. Therefore, our findings indicate that NF-кB-S468 phosphorylation may be relevant to dentate astroglial loss.

\section{Effects of Kinase Inhibitors, PARP1 Inhibitor and AKT Activator on Astroglial Viability Following SE}

Next, we evaluated the effects of kinase inhibitors on astroglial viability in response to SE. TUNEL positive astrocytes were rarely detected in CA1 astrocytes following SE (Figures 6A,B). 
3CAI increased the number of TUNEL positive astrocytes in the CA1 region ( $p<0.05$ vs. vehicle, one-way ANOVA, $n=7$, respectively; Figures $\mathbf{6 A , B}$ ), while BIM (Figures $\mathbf{6 A , B}$ ), U0126 and KN-93 (data not shown) did not affect it. To directly elucidate the roles of PARP1 and AKT in astroglial viability, we applied PJ-34 (a PARP1 inhibitor) and SC79 (an AKT activator). Both PJ-34 and SC79 did not influence the number of TUNEL positive astrocytes in the CA1 region (Figures $\mathbf{6 A , B}$ ). In contrast to CA1 astrocytes, SE significantly increased the number of TUNEL positivity in dentate astrocytes $(p<0.05$ vs. control animals, one-way ANOVA, $n=7$, respectively; Figures 6A,C). 3CAI, PJ-34 and U0126 (data not shown) elevated the number of TUNEL positive astrocytes in this region ( $p<0.05$ vs. vehicle, one-way ANOVA, $n=7$, respectively; Figures 6A,C), while BIM (Figures 6A,C) and KN-93 (data not shown) did not affect it. However, SC79 effectively diminished the number of TUNELpositive dentate astrocytes following SE $(p<0.05$ vs. vehicle, one-way ANOVA, $n=7$, respectively; Figures 6A,C). These findings indicate that PARP1, ERK1/2 and AKT may be involved in astroglial viability in response to SE.

\section{DISCUSSION}

PARP1 plays a different role in reactive astrogliosis and astroglial apoptosis following SE: PARP1 degradation/inhibition evokes astroglial death, but its activation results in reactive astrogliosis (Kim et al., 2014). Although DNA damage is a general cause of PARP1 activation, DNA damage-independent PARP1 activation has been also reported (Kauppinen et al., 2006; Spina-Purrello et al., 2008). Therefore, it is likely that other regulatory signaling molecules for PARP1 activation beyond DNA damage may be involved in the distinct astroglial response to $\mathrm{SE}$, albeit at a lesser extent.

Consistent with our previous study (Kim et al., 2014), the present data show that SE elevated PARP1 expression and PAR level in CA1 reactive astrocytes, which are indicatives of PARP1 activation. Since PARP1 regulates cell proliferation and inflammatory responses via various transcription factors such as NF- $\mathrm{B}$, activator protein-1 and cAMP-response element binding protein (Ha et al., 2002; Hassa et al., 2003), our findings indicate that PARP1 activation may play an important role in $\mathrm{CA} 1$ reactive astrogliosis. On the other hand, pilocarpine increases PARP1 expression in CA1 astrocytes of acute brain slice model (Kim et al., 2014). Furthermore, the astroglial proliferation induced by SE is predominantly observed in the CA1 region at 1 week after SE (Kim and Kang, 2018). Taken together, it is likely that PARP1 expression may be up-regulated in naïve CA1 astrocytes rather than newly generated astrocytes.

The present study also reveals that BIM, 3CAI and U0126, but not KN-93, effectively mitigated SE-induced PARP1 activation in CA1 astrocytes. Indeed, PKC increases PARP1 activity in various cell types (Henderson et al., 2017), although PKC-mediated PARP1 phosphorylation protects from DNA damage-induced necrotic cell death (Hegedus et al., 2008). Furthermore, ERK1/2 activates PARP1 is independent of DNA damage (Cohen-Armon et al., 2007), which is required for maximal PARP1 activation (Kauppinen et al., 2006) AKT also regulates PARP1 cleavage-mediated apoptosis (Chiarugi, 2002). With respect to these previous studies, our findings suggest that PKC, ERK1/2 and AKT, but not CaMKII, may modulate PARP1mediated CA1 reactive astrogliosis. In contrast, down-regulation of PARP1 expression was observed in dentate astrocytes following SE. U0126 and 3CAI aggravated dentate astroglial loss concomitant with decreasing PARP1 expression. In addition, SC79 effectively abrogated apoptosis of dentate astrocytes, while PJ-34 deteriorated it. Thus, our findings indicate that AKT- and ERK1/2-mediated PARP1 activation may play an important role in the viability of dentate astrocytes following SE.

In the present study, BIM reduced PARP1 expression and PAR synthesis level, but U0126 only reduced the PAR level following SE. Although we could not exactly explain these discrepancies in the present study, it is considerable that the different mechanisms/efficacies of BIM and U0126 to inhibit reactive astrogliosis would distinctly affect PARP1 expression and PAR synthesis. This is because $\mathrm{PKC}$ regulates reactive astrogliosis and astroglial proliferation (Stanimirovic et al., 1995; Scarisbrick et al., 2012), while ERK1/2 activation is insufficient to induce astroglial proliferation during the process of reactive astrogliosis (Kim and Kang, 2018). Therefore, the differential effects of BIM and U0126 on PARP1 expression and PAR synthesis may result from the distinct underlying mechanisms of BIM and U0126 for inhibiting reactive astrogliosis.

Recently, we have reported that the increased PKC-mediated PEA15-S104 phosphorylation plays an important role in reactive CA1 astrogliosis, while the reduced PEA15-S116 phosphorylation is relevant to apoptosis of dentate astrocytes induced by SE, independent of neuronal damage (Park and Kang, 2018). In the present study, PEA15-S104, but not -S116, phosphorylation was up-regulated in reactive CA1 astrocytes following SE, which was down-regulated by BIM. Since BIM attenuated reactive $\mathrm{CA} 1$ astrogliosis induced by SE, it is likely that PEA15-S104 phosphorylation may be involved in reactive astrogliosis. However, 3CAI and U0126 mitigated reactive CA1 astrogliosis concomitant with reducing PARP1 activity, which did not affect PEA15-S104 phosphorylation. Therefore, our findings suggest that PARP1 activation may play a more important role in reactive astrogliosis than PEA15-S104 phosphorylation. The present study also demonstrates that SE diminished PEA15-S116 phosphorylation in dentate astrocytes. BIM, 3CAI, U0126 and KN-93 did not influence PEA15-S116 phosphorylation in dentate astrocytes. However, 3CAI led to CA1 astroglial apoptosis and deteriorated it in dentate astrocytes following SE, which were abrogated by SC79. Considering that PEA15 phosphorylations influence cell viability and PEA15 stability by inhibiting apoptosis and proteasomal degradation (Danziger et al., 1995; Kubes et al., 1998; Trencia et al., 2003; Perfetti et al., 2007), our findings suggest that AKT-mediated PEA15-S116 phosphorylation may play a pro-survival role in astrocytes against apoptosis.

NF-кB phosphorylations transactivate several anti-apoptotic and pro-survival genes (Chiarugi, 2002; Liu et al., 2012). In addition, muscarinic receptor (receptor for pilocarpine) modulates NF- $\kappa$ B translocations and its phosphorylations in astrocytes (Guizzetti et al., 2003), which regulate reactive 
astrogliosis (Morga et al., 2009). In the present study, SE increased NF- $\mathrm{B}-\mathrm{S} 311$ phosphorylation in CA1 astrocytes, which was mitigated by BIM, 3CAI and U0126. These findings indicate that PKC-, AKT- and ERK1/2-mediated signaling pathways may regulate NF- $\kappa \mathrm{B}-\mathrm{S} 311$ phosphorylation during reactive astrogliosis. The present data also reveal that $\mathrm{SE}$ elevated NF- $\kappa \mathrm{B}-\mathrm{S} 468$ phosphorylation in CA1 astrocytes, which was unaffected by BIM, 3CAI, U0126 and KN-93. Since the phosphorylation of $\mathrm{S} 468$ site terminates NF- $\mathrm{B}$ dependent gene expression upon assisting in binding of an E3 ubiquitin ligase complex to $\mathrm{NF}-\kappa \mathrm{B}$, which modulates the removal of chromatin-bound NF- $\mathrm{NB}$ at promoter sites of a subset of NF- $\kappa B$ genes (Geng et al., 2009; Mao et al., 2009), it is likely that the increased NF- $\mathrm{BB}-\mathrm{S} 468$ phosphorylation in CA1 astrocytes may be an adaptive response to inhibit reactive astrogliosis. In dentate astrocytes, however, 3CAI and U0126 enhanced the up-regulation of NF- $\kappa \mathrm{B}-\mathrm{S} 468$ phosphorylation induced by SE. Both AKT and ERK1/2 inhibit glycogen synthase kinase $3 \beta$ (Cohen and Frame, 2001; Lin et al., 2011), which diminishes NF-кB-S468 phosphorylation (Geng et al., 2009; Mao et al., 2009). In addition, NF-кB inhibition triggers rapid PAPR1 cleavage and subsequent apoptosis (Chiarugi, 2002). Thus, our findings suggest that the NF- $\mathrm{BB}-\mathrm{S} 468$ phosphorylation may play a pro-apoptotic role in dentate astrocytes.

In the present study, various kinase inhibitors affected activities of PARP1, PEA15 and NF- $\kappa$ B in astrocytes following SE. Since PJ-34 decreases PARP1 and NF- $\mathrm{B}$ expressions independent of DNA damage (Spina-Purrello et al., 2008; Wang et al., 2013), PARP1 expression is positively correlated with NF- $\mathrm{B}$ expression. Indeed, PARP1 directly binds and interacts with NFKB in cellular level (Stanisavljevic et al., 2011), which are regulated by various mechanisms: PARP1 inhibitors reduce $\mathrm{NF}-\kappa \mathrm{B}$ activity by preventing the degradation of I $\mathrm{B}$ (Genovese et al., 2005; Stilmann et al., 2009). In addition, PARP1-NF- $\mathrm{B}$ interactions synergistically activate transcriptional factors independent of enzymatic activity and DNA-binding ability (Hassa et al., 2003). Therefore, it is likely that PARP1 may interact with NF- $\kappa \mathrm{B}$ to increase NF- $\kappa \mathrm{B}$ activity, which in turn may modulate NF- $\kappa \mathrm{B}$-dependent

\section{REFERENCES}

Anderson, C. M., and Swanson, R. A. (2000). Astrocyte glutamate transport: review of properties, regulation, and physiological functions. Glia 32, 1-14. doi: 10.1002/1098-1136(200010)32:1<1::aid-glia10 $>3.3 . \operatorname{co} ; 2-\mathrm{n}$

Araujo, H., Danziger, N., Cordier, J., Glowinski, J., and Chneiweiss, H. (1993). Characterization of PEA-15, a major substrate for protein kinase C in astrocytes. J. Biol. Chem. 268, 5911-5920.

Chiarugi, A. (2002). Characterization of the molecular events following impairment of NF-kappaB-driven transcription in neurons. Mol. Brain Res. 109, 179-188. doi: 10.1016/s0169-328x(02)00558-2

Cohen, P., and Frame, S. (2001). The renaissance of GSK3. Nat. Rev. Mol. Cell Biol. 2, 769-776. doi: 10.1038/35096075

Cohen-Armon, M., Visochek, L., Rozensal, D., Kalal, A., Geistrikh, I., Klein, R., et al. (2007). DNA-independent PARP-1 activation by phosphorylated ERK2 increases Elk1 activity: a link to histone acetylation. Mol. Cell 25, 297-308. doi: 10.1016/j.molcel.2006.12.012 gene expression. PEA15 is also involved in NF- $\mathrm{B}$-dependent transcriptions (Wakita et al., 2016) and regulates PARP1 activity via nuclear ERK1/2 translocation (Krueger et al., 2005; SpinaPurrello et al., 2008). With respect to these reports, it is presumable that PARP1-PEA15-NF-кB-mediated framework would differently regulate the regional specific astroglial responses following SE, although it was not directly confirmed in the present study. Further studies are needed to elucidate this hypothesis.

In conclusion, the present study demonstrates that PKC, AKT and ERK1/2 differently regulated activities of PARP1, PEA15 and NF-кB in CA1 astrocytes and dentate astrocytes. Therefore, our findings suggest that the protein kinases may be distinctly involved in regional specific astroglial responses to $\mathrm{SE}$ through various signaling molecules, which would hypothesize their networks to regulate astroglial responses to SE.

\section{DATA AVAILABILITY}

All datasets generated for this study are included in the manuscript.

\section{ETHICS STATEMENT}

All animal experimental procedures and protocols were approved by the Institutional Animal Care and Use Committee of the Hallym University (Chuncheon, South Korea).

\section{AUTHOR CONTRIBUTIONS}

J-EK and T-CK designed the project, performed the experiments described in the manuscript, analyzed the data, and wrote the manuscript.

\section{FUNDING}

This study was supported by a grant of National Research Foundation of South Korea (NRF; No. 2018R1A2A2A05018222 and 2018R1C1B6005216).

Danziger, N., Yokoyama, M., Jay, T., Cordier, J., Glowinski, J., Chneiweiss, H., et al. (1995). Cellular expression, developmental regulation and phylogenic conservation of PEA-15, the astrocytic major phosphoprotein and protein kinase C substrate. J. Neurochem. 64, 1016-1025. doi: 10.1046/j.1471-4159. 1995.64031016.x

Estellés, A., Charlton, C. A., and Blau, H. M. (1999). The phosphoprotein protein PEA-15 inhibits Fas- but increases TNF-R1-mediated caspase- 8 activity and apoptosis. Dev. Biol. 216, 16-28. doi: 10.1006/dbio.1999.9510

Estellés, A., Yokoyama, M., Nothias, F., Vincent, J. D., Glowinski, J., Vernier, P., et al. (1996). The major astrocytic phosphoprotein PEA-15 is encoded by two mRNAs conserved on their full length in mouse and human. J. Biol. Chem. 271, 14800-14806. doi: 10.1074/jbc.271.25.14800

Geng, H., Wittwer, T., Dittrich-Breiholz, O., Kracht, M., and Schmitz, M. L. (2009) Phosphorylation of NF- $\kappa$ B p65 at Ser468 controls its COMMD1-dependent ubiquitination and target gene-specific proteasomal elimination. EMBO Rep. 10, 381-386. doi: 10.1038/embor.2009.10

Genovese, T., Mazzon, E., Muià, C., Patel, N. S., Threadgill, M. D., Bramanti, P., et al. (2005). Inhibitors of poly (ADP-ribose) polymerase modulate signal 
transduction pathways and secondary damage in experimental spinal cord trauma. J. Pharmacol. Exp. Ther. 312, 449-457. doi: 10.1124/jpet.104.076711

Gerace, E., Scartabelli, T., Formentini, L., Landucci, E., Moroni, F., Chiarugi, A., et al. (2012). Mild activation of poly(ADP-ribose) polymerase (PARP) is neuroprotective in rat hippocampal slice models of ischemic tolerance. Eur. J. Neurosci. 36, 1993-2005. doi: 10.1111/j.1460-9568.2012.08116.x

Goebel, D. J. (2009). Selective blockade of CaMKII- $\alpha$ inhibits NMDA-induced caspase-3-dependent cell death but does not arrest PARP-1 activation or loss of plasma membrane selectivity in rat retinal neurons. Brain Res. 1256, 190-204. doi: 10.1016/j.brainres.2008.12.051

Guizzetti, M., Bordi, F., Dieguez-Acuña, F. J., Vitalone, A., Madia, F., Woods, J. S., et al. (2003). Nuclear factor kappaB activation by muscarinic receptors in astroglial cells: effect of ethanol. Neuroscience 120, 941-950. doi: 10.1016/s0306-4522(03)00401-9

Ha, H. C., Hester, L. D., and Snyder, S. H. (2002). Poly(ADP-ribose) polymerase-1 dependence of stress-induced transcription factors and associated gene expression in glia. Proc. Natl. Acad. Sci. U S A 99, 3270-3275. doi: 10.1073/pnas. 052712399

Ha, H. C., and Snyder, S. H. (1999). Poly(ADP-ribose) polymerase is a mediator of necrotic cell death by ATP depletion. Proc. Natl. Acad. Sci. U S A 96, 13978-13982. doi: 10.1073/pnas.96.24.13978

Hassa, P. O., Buerki, C., Lombardi, C., Imhof, R., and Hottiger, M. O. (2003). Transcriptional coactivation of nuclear factor-kappaB-dependent gene expression by $\mathrm{p} 300$ is regulated by poly(ADP)-ribose polymerase-1. J. Biol. Chem. 278, 45145-45153. doi: 10.1074/jbc.m307957200

Hegedus, C., Lakatos, P., Oláh, G., Tóth, B. I., Gergely, S., Szabó, E., et al. (2008). Protein kinase $\mathrm{C}$ protects from DNA damage-induced necrotic cell death by inhibiting poly(ADP-ribose) polymerase-1. FEBS Lett. 582, 1672-1678. doi: 10.1016/j.febslet.2008.04.023

Henderson, D. J. P., Miranda, J. L., and Emerson, B. M. (2017). The $\beta-\mathrm{NAD}^{+}$ salvage pathway and PKC-mediated signaling influence localized PARP-1 activity and CTCF Poly(ADP)ribosylation. Oncotarget 8, 64698-64713. doi: $10.18632 /$ oncotarget.19841

Horner, P. J., and Gage, F. H. (2000). Regenerating the damaged central nervous system. Nature 407, 963-970. doi: 10.1038/35039559

Kang, T. C., Kim, D. S., Kwak, S. E., Kim, J. E., Won, M. H., and Kim, D. W. (2006). Epileptogenic roles of astroglial death and regeneration in the dentate gyrus of experimental temporal lobe epilepsy. Glia 54, 258-271. doi: 10.1002/glia.20380

Kasischke, K. A., Vishwasrao, H. D., Fisher, P. J., Zipfel, W. R., and Webb, W. W. (2004). Neural activity triggers neuronal oxidative metabolism followed by astrocytic glycolysis. Science 305, 99-103. doi: 10.1126/science.1096485

Kaufmann, S. H., Desnoyers, S., Ottaviano, Y., Davidson, N. E., and Poirier, G. G. (1993). Specific proteolytic cleavage of poly(ADP-ribose) polymerase: an early marker of chemotherapy-induced apoptosis. Cancer Res. 53, 3976-3985.

Kauppinen, T. M., Chan, W. Y., Suh, S. W., Wiggins, A. K., Huang, E. J., and Swanson, R. A. (2006). Direct phosphorylation and regulation of poly(ADPribose) polymerase-1 by extracellular signal-regulated kinases 1/2. Proc. Natl. Acad. Sci. U S A 103, 7136-7141. doi: 10.1073/pnas.0508606103

Kim, J. E., Hyun, H. W., Min, S. J., and Kang, T. C. (2017). Sustained HSP25 expression induces clasmatodendrosis via ER stress in the rat hippocampus. Front. Cell. Neurosci. 11:47. doi: 10.3389/fncel.2017.00047

Kim, J. E., and Kang, T. C. (2018). Nucleocytoplasmic p2 $27^{\text {Kip } 1}$ export is required for ERK1/2-mediated reactive astroglial proliferation following status epilepticus. Front. Cell. Neurosci. 12:152. doi: 10.3389/fncel.2018.00152

Kim, J. E., Kim, Y. J., Kim, J. Y., and Kang, T. C. (2014). PARP1 activation/expression modulates regional-specific neuronal and glial responses to seizure in a hemodynamic-independent manner. Cell Death. Dis. 5:e1362. doi: 10.1038/cddis.2014.331

Kim, D. S., Kim, J. E., Kwak, S. E., Choi, K. C., Kim, D. W., Kwon, O. S., et al. (2008). Spatiotemporal characteristics of astroglial death in the rat hippocampo-entorhinal complex following pilocarpine-induced status epilepticus. J. Comp. Neurol. 511, 581-598. doi: 10.1002/cne.21851

Kim, J. E., Kim, D. S., Ryu, H. J., Kim, W. I., Kim, M. J., Kim, D. W., et al. (2013). The effect of P2X7 receptor activation on nuclear factor- $\mathrm{KB}$ phosphorylation induced by status epilepticus in the rat hippocampus. Hippocampus 23, 500-514. doi: 10.1002/hipo.22109

Kitsberg, D., Formstecher, E., Fauquet, M., Kubes, M., Cordier, J., Canton, B., et al. (1999). Knock-out of the neural death effector domain protein PEA-15 demonstrates that its expression protects astrocytes from TNF $\alpha$-induced apoptosis. J. Neurosci. 19, 8244-8251. doi: 10.1523/JNEUROSCI.19-19-08244. 1999

Krueger, J., Chou, F. L., Glading, A., Schaefer, E., and Ginsberg, M. H. (2005). Phosphorylation of phosphoprotein enriched in astrocytes (PEA-15) regulates extracellular signal-regulated kinase-dependent transcription and cell proliferation. Mol. Biol. Cell 16, 3552-3561. doi: 10.1091/mbc.e04-11-1007

Kubes, M., Cordier, J., Glowinski, J., Girault, J. A., and Chneiweiss, H. (1998). Endothelin induces a calcium-dependent phosphorylation of PEA-15 in intact astrocytes: identification of Ser104 and Ser116 phosphorylated, respectively, by protein kinase $\mathrm{C}$ and calcium/calmodulin kinase II in vitro. J. Neurochem. 71, 1307-1314. doi: 10.1046/j.1471-4159.1998.71031307.x

Lazebnik, Y. A., Kaufmann, S. H., Desnoyers, S., Poirier, G. G., and Earnshaw, W. C. (1994). Cleavage of poly(ADP-ribose) polymerase by a proteinase with properties like ICE. Nature 371, 346-347. doi: $10.1038 / 371346 \mathrm{a} 0$

Lin, C. L., Tseng, H. C., Chen, R. F., Chen, W. P., Su, M. J., Fang, K. M., et al.

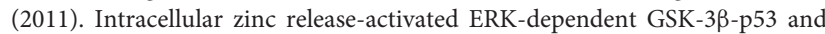
Noxa-Mcl-1 signaling are both involved in cardiac ischemic-reperfusion injury. Cell Death Differ. 18, 1651-1663. doi: 10.1038/cdd.2011.80

Liu, L., Ke, Y., Jiang, X., He, F., Pan, L., Xu, L., et al. (2012). Lipopolysaccharide activates ERK-PARP-1-RelA pathway and promotes nuclear factor- $\kappa \mathrm{B}$ transcription in murine macrophages. Hum. Immunol. 73, 439-447. doi: 10.1016/j.humimm.2012.02.002

Liu, X., Yan, S., Zhou, T., Terada, Y., and Erikson, R. L. (2004). The MAP kinase pathway is required for entry into mitosis and cell survival. Oncogene 23 , 763-776. doi: 10.1038/sj.onc. 1207188

Mao, X., Gluck, N., Li, D., Maine, G. N., Li, H., Zaidi, I. W., et al. (2009). GCN5 is a required cofactor for a ubiquitin ligase that targets NF-kappaB/RelA. Genes Dev. 23, 849-861. doi: 10.1101/gad.1748409

Mazzanti, M., Sul, J. Y., and Haydon, P. G. (2001). Glutamate on demand: astrocytes as a ready source. Neuroscientist 7, 396-405. doi: $10.1177 / 107385840100700509$

Meini, A., Sticozzi, C., Massai, L., and Palmi, M. (2008). A nitric oxide/ $\mathrm{Ca}^{2+} /$ calmodulin/ERK1/2 mitogen-activated protein kinase pathway is involved in the mitogenic effect of IL-1 $\beta$ in human astrocytoma cells. $B r$. J. Pharmacol. 153, 1706-1717. doi: 10.1038/bjp.2008.40

Midorikawa, R., Takei, Y., and Hirokawa, N. (2006). KIF4 motor regulates activitydependent neuronal survival by suppressing PARP-1 enzymatic activity. Cell 125, 371-383. doi: 10.1016/j.cell.2006.02.039

Mizuguchi, H., Terao, T., Kitai, M., Ikeda, M., Yoshimura, Y., Das, A. K., et al. (2011). Involvement of protein kinase $\mathrm{C} \delta /$ extracellular signalregulated kinase/poly(ADP-ribose) polymerase-1 (PARP-1) signaling pathway in histamine-induced up-regulation of histamine $\mathrm{H} 1$ receptor gene expression in HeLa cells. J. Biol. Chem. 286, 30542-30551. doi: 10.1074/jbc.m111.253104

Morga, E., Mouad-Amazzal, L., Felten, P., Heurtaux, T., Moro, M., Michelucci, A., et al. (2009). Jagged1 regulates the activation of astrocytes via modulation of NFKB and JAK/STAT/SOCS pathways. Glia 57, 1741-1753. doi: 10.1002/glia. 20887

Panickar, K. S., and Norenberg, M. D. (2005). Astrocytes in cerebral ischemic injury: morphological and general considerations. Glia 50, 287-298. doi: 10.1002/glia.20181

Park, J. Y., and Kang, T. C. (2018). The differential roles of PEA15 phosphorylations in reactive astrogliosis and astroglial apoptosis following status epilepticus. Neurosci. Res. 137, 11-22. doi: 10.1016/j.neures. 2018.02.003

Perfetti, A., Oriente, F., Iovino, S., Alberobello, A. T., Barbagallo, A. P., Esposito, I., et al. (2007). Phorbol esters induce intracellular accumulation of the anti-apoptotic protein PED/PEA-15 by preventing ubiquitinylation and proteasomal degradation. J. Biol. Chem. 282, 8648-8657. doi: 10.1074/jbc. m608359200

Rossi, D. J., Brady, J. D., and Morh, C. (2007). Astrocyte metabolism and signaling during brain ischemia. Nat. Neurosci. 10, 1377-1386. doi: 10.1038/nn2004

Scarisbrick, I. A., Radulovic, M., Burda, J. E., Larson, N., Blaber, S. I., Giannini, C., et al. (2012). Kallikrein 6 is a novel molecular trigger of reactive astrogliosis. Biol. Chem. 393, 355-367. doi: 10.1515/hsz-2011-0241

Schmidt-Kastner, R., and Ingvar, M. (1994). Loss of immunoreactivity for glial fibrillary acidic protein (GFAP) in astrocytes as a marker for profound tissue 
damage in substantia nigra and basal cortical areas after status epilepticus induced by pilocarpine in rat. Glia 12, 165-172. doi: 10.1002/glia.4401 20302

Schmidt-Kastner, R., and Ingvar, M. (1996). Laminar damage of neurons and astrocytes in neocortex and hippocampus of rat after long-lasting status epilepticus induced by pilocarpine. Epilepsy Res. Suppl. 12, 309-316.

Shibuya, M. (2009). Brain angiogenesis in developmental and pathological processes: therapeutic aspects of vascular endothelial growth factor. FEBS J. 276, 4636-4643. doi: 10.1111/j.1742-4658.2009.07175.x

Simard, M., and Nedergaard, M. (2004). The neurobiology of glia in the context of water and ion homeostasis. Neuroscience 129, 877-896. doi: 10.1016/j. neuroscience.2004.09.053

Spina-Purrello, V., Patti, D., Giuffrida-Stella, A. M., and Nicoletti, V. G. (2008). Parp and cell death or protection in rat primary astroglial cell cultures under LPS/IFN $\gamma$ induced proinflammatory conditions. Neurochem. Res. 33, 2583-2592. doi: 10.1007/s11064-008-9835-1

Stanimirovic, D. B., Ball, R., Mealing, G., Morley, P., and Durkin, J. P. (1995). The role of intracellular calcium and protein kinase $\mathrm{C}$ in endothelin-stimulated proliferation of rat type I astrocytes. Glia 15, 119-130. doi: 10.1002/glia. 440150204

Stanisavljevic, J., Porta-de-la-Riva, M., Batlle, R., de Herreros, A. G., and Baulida, J. (2011). The p65 subunit of NF-кB and PARP1 assist Snaill in activating fibronectin transcription. J. Cell Sci. 124, 4161-4171. doi: 10.1242/jcs. 078824

Stilmann, M., Hinz, M., Arslan, S. C., Zimmer, A., Schreiber, V., Scheidereit, C. (2009). A nuclear poly (ADP-ribose)-dependent signalosome confers DNA damage-induced IкB kinase activation. Mol. Cell 36, 365-378. doi: 10.1016/j. molcel.2009.09.032

Takano, T., Tian, G. F., Peng, W., Lou, N., Libionka, W., Han, X., et al. (2006). Astrocyte-mediated control of cerebral blood flow. Nat. Neurosci. 9, 260-267. doi: $10.1038 / \mathrm{nn} 1623$
Trencia, A., Perfetti, A., Cassese, A., Vigliotta, G., Miele, C., Oriente, F., et al. (2003). Protein kinase B/Akt binds and phosphorylates PED/PEA15, stabilizing its antiapoptotic action. Mol. Cell. Biol. 23, 4511-4521. doi: $10.1128 / \mathrm{mcb} .23 .13 .4511-4521.2003$

Viatour, P., Merville, M. P., Bours, V., and Chariot, A. (2005). Phosphorylation of NF-кB and IкB proteins: implications in cancer and inflammation. Trends Biochem. Sci. 30, 43-52. doi: 10.1016/j.tibs.2004.11.009

Wakita, M., Edamatsu, H., Li, M., Emi, A., Kitazawa, S., and Kataoka, T. (2016). Phospholipase $\mathrm{C} \epsilon$ activates nuclear factor- $\mathrm{\kappa B}$ signaling by causing cytoplasmic localization of ribosomal S6 kinase and facilitating its phosphorylation of inhibitor $\kappa \mathrm{B}$ in colon epithelial cells. J. Biol. Chem. 291, 12586-12600. doi: 10.1074/jbc.M116.717561

Wang, Z., Li, Y., Lv, S., and Tian, Y. (2013). Inhibition of proliferation and invasiveness of ovarian cancer $\mathrm{C} 13^{*}$ cells by a poly(ADP-ribose) polymerase inhibitor and the role of nuclear factor-кB. J. Int. Med. Res. 41, 1577-1585. doi: 10.1177/0300060513480913

Ying, W., Chen, Y., Alano, C. C., and Swanson, R. A. (2002). Tricarboxylic acid cycle substrates prevent PARP-mediated death of neurons and astrocytes. J. Cereb. Blood Flow Metab. 22, 774-779. doi: 10.1097/00004647-20020700000002

Conflict of Interest Statement: The authors declare that the research was conducted in the absence of any commercial or financial relationships that could be construed as a potential conflict of interest.

Copyright (C) 2019 Kim and Kang. This is an open-access article distributed under the terms of the Creative Commons Attribution License (CC BY). The use, distribution or reproduction in other forums is permitted, provided the original author(s) and the copyright owner(s) are credited and that the original publication in this journal is cited, in accordance with accepted academic practice. No use, distribution or reproduction is permitted which does not comply with these terms. 